\title{
Quantification of Pharmaceutical Polymorphs and Prediction of Dissolution Rate Using Theophylline Tablet by Terahertz Spectroscopy
}

\author{
Jin Hisazumi, ${ }^{* a}$ Tatsuya Suzuki, ${ }^{a}$ Hiroaki Nakagami, ${ }^{a}$ and Katsuhide TeradA ${ }^{b}$ \\ ${ }^{a}$ Formulation Technology Research Laboratories, Pharmaceutical Technology Division, Daiichi Sankyo Co., Ltd.; 1-12-1 \\ Shinomiya, Hiratsuka, Kanagawa 254-0014, Japan: and ${ }^{b}$ Faculty of Pharmaceutical Sciences, Toho University; 2-2-1 \\ Miyama, Funabashi, Chiba 274-8510, Japan. \\ Received November 5, 2010; accepted January 28, 2011; published online January 28, 2011
}

Theophylline has an anhydrous form and a monohydrated form, and the dissolution rate of the anhydrous form is higher than that of the monohydrated form. Terahertz (THz) spectra of theophylline tablet containing the theophylline anhydrous form, monohydrated form, microcrystalline cellulose and magnesium stearate exhibited a specific absorption peak at $0.96 \mathrm{THz}$, where the theophylline anhydrous form demonstrated an absorption peak. Additionally, the intensity of the peak at $0.96 \mathrm{THz}$ gradually decreased as the proportion of the anhydrous form decreased. The multivariate data analysis was performed to correlate the $\mathrm{THz}$ spectra of theophylline tablets with the ratio of the theophylline anhydrous form. The calibration model used to predict the mixing ratio of the theophylline anhydrous form from the THz spectra achieved root-mean-squared errors of cross-validation (RMSECV) of $2.89 \%$, a slope of 0.9934 and an $R^{2}$ of 0.9927 . In addition, there were intentions to develop a prediction model for the dissolution rate of theophylline from the drug product. The dissolution rate of theophylline tablet was gradually delayed as the proportion of the anhydrous form was decreased. The multivariate data analysis was performed to correlate the $\mathrm{THz}$ spectra of theophylline tablets with the dissolution rate. The calibration model used to predict the percentage of theophylline dissolved in $45 \mathrm{~min}$ from the $\mathrm{THz}$ spectra achieved an RMSECV of $3.29 \%$, a slope of 0.9260 and an $R^{2}$ of 0.9423 . Furthermore, there were no significant differences between the predicted and measured percentages of theophylline dissolved in $45 \mathrm{~min}$ in the theophylline tablets that were stored at $84 \%$ relative humidity $(\mathrm{RH})$ and $25^{\circ} \mathrm{C}$ for $12 \mathrm{~h}$ or $3 \mathrm{~d}$.

Key words terahertz spectroscopy; polymorph; theophylline; multivariate data analysis; quantification; dissolution

It is well known that many pharmaceutical solids can exist in more than one crystalline form as polymorphism. ${ }^{1)}$ Polymorphism may also include solvation or hydration products (also known as pseudopolymorphs) and is amorphous, ${ }^{2)}$ which and they can lead to different physical and physicochemical properties. These may also impact on the pharmaceutical properties of the drug product, for example, different dissolution rate or bioavailability. ${ }^{3)}$ Crystalline form may change due to the stress in the manufacturing process of the drug product and storage conditions. Therefore, it is useful to detect the polymorphism in the drug product.

Although X-ray powder diffraction method is the most popular technique used for detecting and quantifying polymorphs, recently, vibrational spectroscopic analysis such as Raman spectroscopy and near-infrared (NIR) spectroscopy have been used for the same purpose as non-destructive measuring methods. ${ }^{4-7)}$ Additionally, terahertz (THz) spectroscopy has become popular as a novel technique to determine the crystallinity and polymorphism of drug substance. $^{8-12)}$ On the other hand, evaluation of solid-state polymorphic change in a drug product by $\mathrm{THz}$ spectroscopy has been scarcely studied.

In this study, THz spectroscopy was applied to evaluate the polymorphism of theophylline in the drug product because theophylline has monohydrated and anhydrous forms. ${ }^{13)}$ The objective of this work was to develop a quantitative calibration model for the ratio of the monohydrated and anhydrous forms in the drug product.

Prediction for drug dissolution from tablets using NIR spectroscopy has been reported. ${ }^{1-16)}$ Although these studies were based on an evaluation of physical properties of tablets or quantitative analyses of some excipients, a prediction study for the same purpose on the basis of an evaluation of polymorphic change has been scarcely studied. The wavelengths of NIR spectroscopy are generally $0.8-2.5 \mu \mathrm{m}$ and close to interatomic distances, whereas those of $\mathrm{THz}$ spectroscopy are $33-3300 \mu \mathrm{m}$ and close to intermolecular distances. NIR and $\mathrm{THz}$ spectroscopy should be appropriate to detect interatomic and itermolecular interactions, respectively, and $\mathrm{THz}$ spectorscopy should be more adequate to analyze the polymorphism. ${ }^{8)}$ Thus, in this article, a prediction model for the dissolution rate of theophylline from the drug product containing the theophylline monohydrated and anhydrous forms, which had different dissolution rates, ${ }^{17}$ ) was intended to be developed using the THz spectroscopy.

\section{Experimental}

Materials Theophylline anhydrous form (Sigma-Aldrich Co., U.S.A.) was of reagent grade. Theophylline monohydrated form was recrystallized from distilled water and dried at room temperature. Theophylline anhydrous form and monohydrated form were passed through a $300 \mu \mathrm{m}$ mesh screen. Microcrystalline cellulose (CEOLUS PH 302, Asahi Kasei Chemicals Corporation, Japan), and magnesium stearate (NF NON-BOVINE HyQual, MALLINCKRODT, INC., U.S.A.) were used as received.

Preparation of Material Pellets Materials were mixed with polyethylene powder (Sigma-Aldrich Co., U.S.A.) homogeneously using an agate mortar and pestle. The mixed materials were compressed under a pressure of $10 \mathrm{kN}$ with flat-faced punches in a die with an internal diameter of $9.0 \mathrm{~mm}$ by a hydraulic oil compactor (Riken Power P-1B, Riken Seiki Co., Ltd., Japan).

Preparation of Theophylline Tablets Materials were mixed and compressed in the same method as the preparation of material pellets. The theophylline tablets were preserved in a chamber at $60 \%$ relative humidity $(\mathrm{RH})$ and $25^{\circ} \mathrm{C}$. It was confirmed that the two forms of theophylline did not change under this condition (data not shown).

Storage of Theophylline Tablets The prepared theophylline tablets were placed on a glass plate and stored in a chamber at $84 \% \mathrm{RH}$ and $25^{\circ} \mathrm{C}$. The theophylline anhydrous form gradually changes to the monohydrated 
form in this condition, and this condition is feasible in daily life. After storage for each period, the stored theophylline tablets were preserved in a chamber at $60 \% \mathrm{RH}$ and $25^{\circ} \mathrm{C}$.

X-Ray Powder Diffraction Analysis The theophylline tablets were milled with an agate mortar and pestle. The X-ray powder diffraction (XRPD) patterns of samples were measured with a diffractometer (Rint2200/PC, Rigaku Corporation, Japan) at room temperature. The measurement conditions were as follows: target, $\mathrm{Cu}$; filter, Ni; voltage, $40 \mathrm{kV}$; current, $40 \mathrm{~mA}$; receiving slit, $1.0 \mathrm{~mm}$; and scanning speed $2.000^{\circ} / \mathrm{min}$.

Dissolution Testing All dissolution testings for theophylline tablets were carried out in $900 \mathrm{ml}$ distilled water using a fully automated United States Pharmacopeia (USP) apparatus II dissolution tester system (Type NTR-6000, Toyama Sangyo Co., Ltd., Japan) at $37 \pm 0.5^{\circ} \mathrm{C}$ and $50 \mathrm{rpm}$. The drug release was assayed by UV spectroscopy (UV-1600, Shimadzu Corporation, Japan) at $270 \mathrm{~nm}$ and $350 \mathrm{~nm}$.

Terahertz Spectroscopy The THz spectra of the pellet of the materials or drug products were acquired by using a $\mathrm{THz}$ time-domain spectroscopy (THz-TDS) apparatus (THz-TDS system RT-10000, Tochigi Nikon Corporation, Japan) with the transmission method. The measurement covered the range of $0.2-2 \mathrm{THz}$ with a resolution of $0.04 \mathrm{THz}$.

Multivariate Data Analysis Multivariate data analyses were performed on the THz spectra using OPUS software (version 6.5, Bruker Optics). THz spectra were converted to their second derivatives. The calibration models were calculated using the partial least squares (PLS) algorithm with the concept of cross-validation (one sample removed per cycle). The root-meansquared errors of cross-validation (RMSECV) were determined for each rank. The number of rank used to construct the models was based on the lowest RMSECV without exceeding three, thus ensuring an acceptable complexity of the resulting model.

\section{Results and Discussion}

THz Spectra of Pure Theophylline Figure 1 shows the $\mathrm{THz}$ spectra for the pellets of the theophylline anhydrous form and monohydrated form, and they were consistent with the patterns reported by Liu et al. ${ }^{18)}$ One specific absorption peak was observed at $0.96 \mathrm{THz}$ for the anhydrous form, but this absorption peak was not observed for the monohydrated form. In addition, no significant absorption peaks were ob-

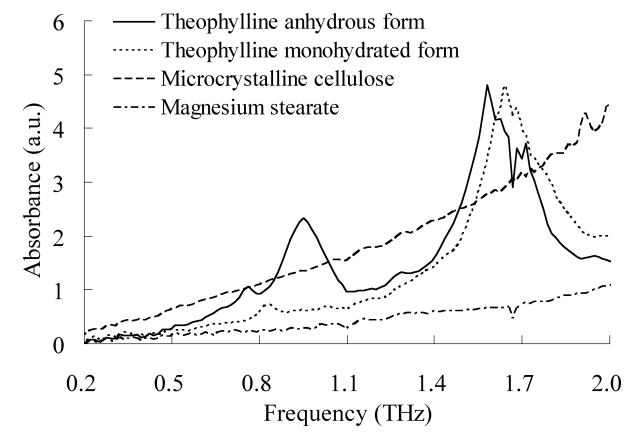

Fig. 1. THz Spectra of Theophylline Anhydrous Form and Monohydrated Form served at $0.2-2.0 \mathrm{THz}$ for microcrystalline cellulose and magnesium stearate.

Prediction for the Mixing Ratio of Theophylline Polymorphs in the Theophylline Tablet The XRPD patterns of theophylline polymorphs and microcrystalline cellulose are shown in Fig. 2. The XRPD patterns of these materials were quite different from each other, and the XRPD patterns of theophylline polymorphs were consistent with the patterns reported by Ono et al. ${ }^{17)}$

The formulae of theophylline tablets are shown in Table 1. The contents of theophylline in the tablets are about $30 \%$. Quantitative analysis of the content of theophylline anhydrous form in a tablet was performed over a content range from $0-25 \%$ using the $\mathrm{THz}$ spectroscopy (data not shown). A quantitation limit of $4.74 \%$ was obtained according to the ICH guideline. ${ }^{19)}$

There were no significant differences between XRPD patterns of blends and tablets (data not shown); therefore, it was confirmed that the polymorphic change of theophylline polymorphs did not occur during the manufacturing of theophylline tablets.

The THz spectra of theophylline tablets are shown in Fig. 3. The $\mathrm{THz}$ spectra of theophylline tablet containing the anhydrous form exhibited a specific absorption peak at $0.96 \mathrm{THz}$. On the other hand, this peak was not observed in the theophylline tablet containing the monohydrated form. The tablets containing the mixtures of theophylline polymorphs showed a combination of this feature; as the proportion of the anhydrous form decreased, the intensity of the peak at $0.96 \mathrm{THz}$ gradually decreased. Therefore, the frequency band around the peak at $0.96 \mathrm{THz}$ should be useful for quantitative analysis of the mixing ratio of theophylline polymorphs in the tablet. Absorption at the range from 1.6 to

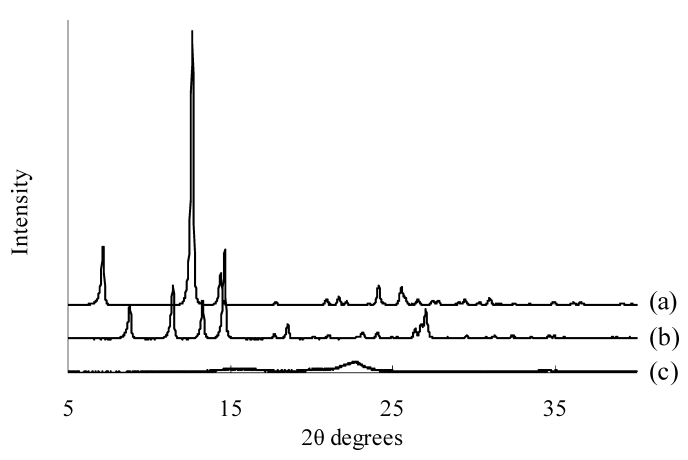

Fig. 2. XRPD Patterns of Pure Materials

(a) Theophylline anhydrous form (b) theophylline monohydrated form (c) microcrystalline cellulose.

Table 1. Formulae of Theophylline Tablets (mg/tablet)

\begin{tabular}{|c|c|c|c|c|c|c|c|}
\hline Tablet No. & 1 & 2 & 3 & 4 & 5 & 6 & 7 \\
\hline Mixing ratio of theophylline anhydrous form ${ }^{a}$ ) & $100 \%$ & $90 \%$ & $80 \%$ & $75 \%$ & $50 \%$ & $25 \%$ & $0 \%$ \\
\hline Theophylline anhydrous form & 35 & 31.5 & 28 & 26.25 & 17.5 & 8.75 & - \\
\hline Theophylline monohydrated form & - & 3.85 & 7.7 & 9.625 & 19.25 & 28.875 & 38.5 \\
\hline Microcrystalline cellulose & 83.8 & 83.8 & 83.8 & 83.8 & 83.8 & 83.8 & 83.8 \\
\hline Magnesium stearate & 1.2 & 1.2 & 1.2 & 1.2 & 1.2 & 1.2 & 1.2 \\
\hline Unit weight & 120 & 120.35 & 120.7 & 120.875 & 121.75 & 122.625 & 123.5 \\
\hline
\end{tabular}

a) Mixing ratio of the theophylline anhydrous form in the drug substance. The molecular weights of the theophylline anhydrous and monohydrated form were 180 and 198 , respectively, and the amount of the theophylline monohydrated form was corrected with the molecular weights from the amount of the anhydrous form. 


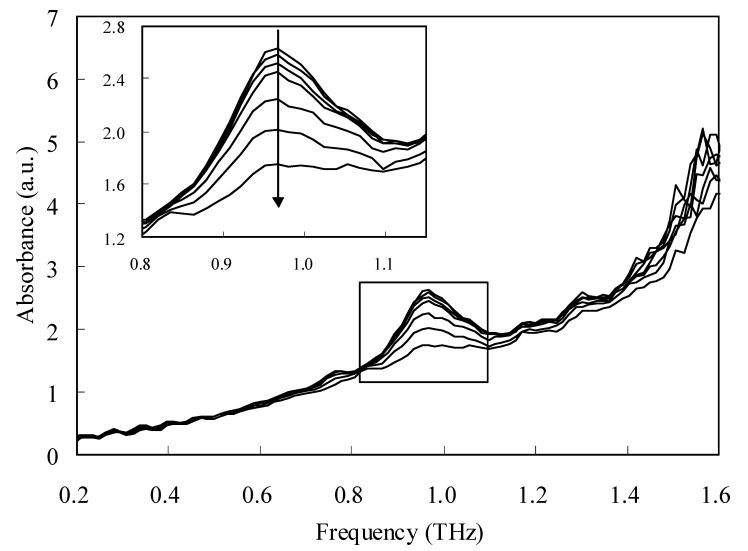

Fig. 3. THz Spectra of Theophylline Tablets Containing Theophylline Polymorphs

Inset: effect of mixing ratio, enhanced view. The arrow indicates the direction of the decreasing ratio of the theophylline anhydrous form.

$2.0 \mathrm{THz}$ was extremely large and the beam hardly transmitted through the tablet. Thus, we showed that the THz spectra was below $1.6 \mathrm{THz}$ in Fig. 3 and we did not use the absorption peak at $1.6 \mathrm{THz}$.

The multivariate data analysis was performed to correlate the THz spectra of theophylline tablets with the mixing ratios of the theophylline anhydrous form. The results of the optimization and performance of statistics for the PLS calibration are summarized in Table 2 and Fig. 4. The calibration model achieved an RMSECV of $2.89 \%$, a slope of 0.9934 and an $R^{2}$ of 0.9927 . These results indicated that the model was a reasonable quantitative model for the ratios of theophylline polymorph mixture in tablets. Furthermore, this suggested that the $\mathrm{THz}$ spectra were useful for quantitative analysis of the mixing ratio of polymorphs in the drug product.

Prediction for the Dissolution Rate of Theophylline Tablet The results of the dissolution testing for the theophylline tablets containing the theophylline mixtures at each ratio are shown in Fig. 5. It was observed that the dissolution rate was gradually delayed as the proportion of the anhydrous form was decreased. In addition, there were no differences in the dissolution profiles of theophylline tablets in which the mixing ratios of the theophylline anhydrous form were 50\% or less. It is suggested that a part of theophylline anhydrous form in the tablet changed to the monohydrated form before dissolution and the other part will be dissolved in the medium directly. The higher the ratio of anhydrous form dissolving directly, the faster the dissolution rate of theophylline tablets. Regarding the theophylline tablets in which the ratio of anhydrous form is from 100 to $75 \%$, the ratio of anhydrous form that dissolves directly will decrease, but the ratio of anhydrous form dissolving through monohydrated form will remain unchanged. Regarding the theophylline tablets in which the ratio of anhydrous form is $50 \%$ or less, almost all of the anhydrous form should change to the monohydrated form before dissolution, and thus there were no difference in the dissolution profiles.

The multivariate data analysis was performed to correlate the percentage dissolved in $45 \mathrm{~min}$ and the $\mathrm{THz}$ spectra of theophylline tablets. The results of the optimization and performance of statistics for the PLS calibration are summarized
Table 2. Performance Parameters of Multivariate Calibration Model for Correlating Theophylline Tablet's THz Spectra with Mixing Ratios of the Theophylline Anhydrous Form

\begin{tabular}{ll}
\hline \hline \multicolumn{1}{c}{ Method } & \multicolumn{1}{c}{ PLS } \\
\hline Frequency band (THz) & $0.9807-0.8051$ \\
Preprocessing & 2nd derivative \\
Rank & 3 \\
RMSECV (\%) & 2.89 \\
\hline
\end{tabular}

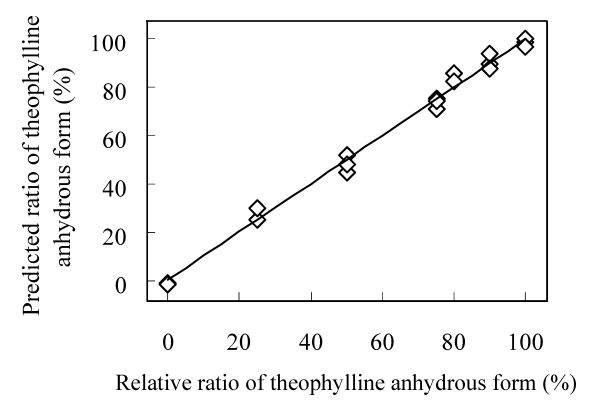

Fig. 4. Multivariate Calibration Model for Correlating Theophylline Tablet's THz Spectra with Mixing Ratios of the Theophylline Anhydrous Form

Line represents linear regression for cross-validation results. The regression equation and $R^{2}$ are as follows: $Y=0.9934 x+0.3550, R^{2}=0.9927$.

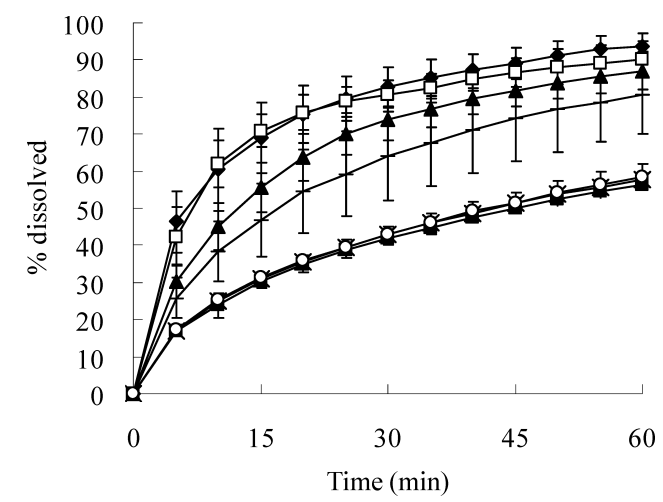

Fig. 5. Results of the Dissolution Testing for Theophylline Tablets Containing Theophylline Polymorphs

Tablet No.: $\bullet, 1 ; \square, 2 ; \boldsymbol{\Lambda}, 3 ;+, 4 ; \times, 5 ; \mathbf{\square}, 6$; $\bigcirc, 7$. Each dissolution profile represents the average value of six dissolution testings (six tablets) and the error bar represents S.D.

Table 3. Performance Parameters of Multivariate Calibration Model for Correlating Percentages Dissolved in 45 min with the THz Spectra of Theophylline Tablets

\begin{tabular}{ll}
\hline \hline \multicolumn{1}{c}{ Method } & \multicolumn{1}{c}{ PLS } \\
\hline Frequency band (THz) & $1.2150-0.9954$ \\
& $0.9515-0.7172$ \\
Preprocessing & 2nd derivative \\
Rank & 1 \\
RMSECV (\%) & 3.29 \\
\hline
\end{tabular}

in Table 3 and Fig. 6. The multivariate data analysis was performed using the data of theophylline tablets containing theophylline mixtures in which proportions of the anhydrous form were not less than $50 \%$. Because there were no differences in the dissolution profiles of tablets in which propor- 


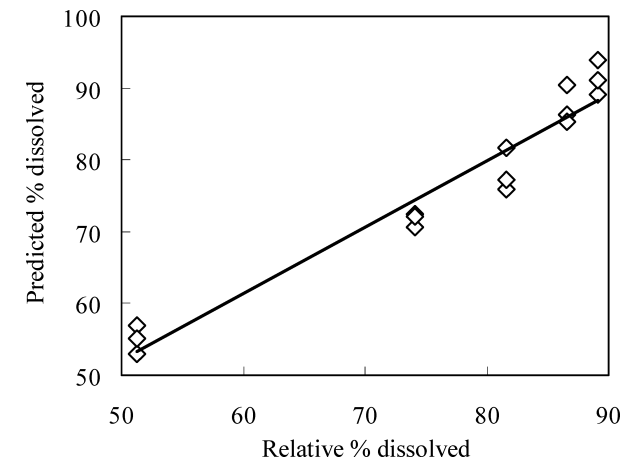

Fig. 6. Multivariate Calibration Model for Correlating Percentages Dissolved in $45 \mathrm{~min}$ with the THz Spectra of Theophylline Tablets

The line represents linear regression for cross-validation results. The regression equation and $R^{2}$ are as follows: $Y=0.9260 x+5.8276, R^{2}=0.9423$. Each reference percentage of dissolved represents the average value of six dissolution testings (six tablets), and three THz spectra (three tablets) were used at each percentage dissolved.

tions of the anhydrous form were $50 \%$ or less, it would not be necessary to analyze these data. The calibration model achieved an RMSECV of $3.29 \%$, a slope of 0.9260 and an $R^{2}$ of 0.9423 ; and the frequency bands were parts of the characteristic peak at $0.96 \mathrm{THz}$ of the theophylline anhydrous form. These results indicated that this model was a reasonable quantitative model for the prediction of the percentage dissolved in $45 \mathrm{~min}$ on the basis of an evaluation of polymorphic change in theophylline tablets. Furthermore, this suggests that the $\mathrm{THz}$ spectra are useful for quantitative analysis for the prediction of the dissolution rate in drug products.

Prediction for the Dissolution Rate of Stored Theophylline Tablet The theophylline tablets containing the theophylline anhydrous form were stored at $84 \% \mathrm{RH}$ and $25^{\circ} \mathrm{C}$. The results of XRPD analysis of the stored samples showed that the anhydrous form transformed to the monohydrated form gradually as the storage period increased (data not shown). The results of the dissolution testing for the stored theophylline tablets are shown in Fig. 7. It was observed that the dissolution rate was gradually delayed as the storage period increased.

The ratio of the theophylline anhydrous form and the percentage dissolved in $45 \mathrm{~min}$ were calculated from the $\mathrm{THz}$ spectra of stored theophylline tablets using the multivariate calibration models. The summary of the predictive values and the measured results of the dissolution testing for stored theophylline tablets are shown in Table 4.

The predicted ratio of the theophylline anhydrous form gradually decreased as the storage period increased, and this result suggests the possibility that the polymorphic change in stored drug products can be quantitatively predicted with $\mathrm{THz}$ spectroscopy. This prediction model for the dissolution ratio is applicable to the theophylline tablets in which the anhydrous form ratio is higher than $50 \%$. The predicted ratio of the theophylline anhydrous form showed a slightly higher value than $50 \%(59.0 \%)$, hence the theophylline tablet was stored until $5 \mathrm{~d}$.

For the results of the dissolution testing, there were no significant differences between the predicted and measured percentages dissolved in $45 \mathrm{~min}$ in the theophylline tablets stored for $12 \mathrm{~h}$ or $3 \mathrm{~d}$. On the other hand, in the theophylline tablets that were stored for $5 \mathrm{~d}$, the measured percentage dissolved in 45 min was higher than the predicted percentage.

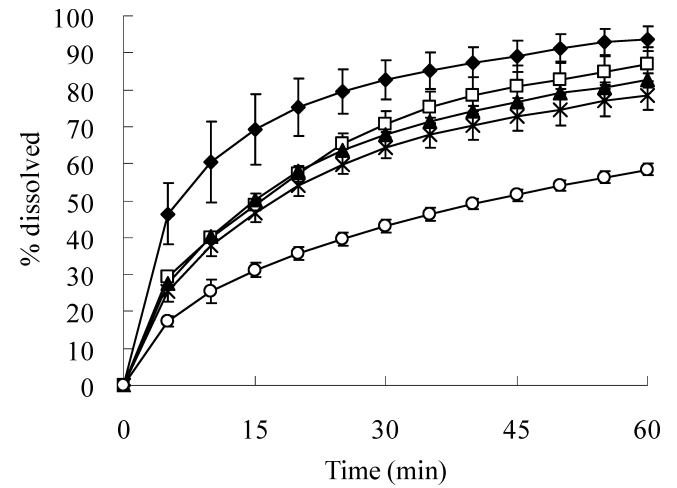

Fig. 7. Results of the Dissolution Testing for Stored Theophylline Tablets

Storage period of theophylline tablet: $\square, 12 \mathrm{~h} ; \boldsymbol{\Delta}, 3 \mathrm{~d}$; $\times, 5 \mathrm{~d}$. Each dissolution profile represents the average value of three dissolution testings (three tablets) and the error bar represents S.D. Data of theophylline tablet containing the theophylline anhydrous form $(\diamond)$ and hydrated form $(\bigcirc)$ were the same as Fig. 5.

Table 4. Ratio of the Theophylline Anhydrous Form and Percentage Dissolved in $45 \mathrm{~min}$ for the Stored Theophylline Tablets $(n=3)$

\begin{tabular}{|c|c|c|c|c|c|c|}
\hline \multirow{3}{*}{$\begin{array}{l}\text { Storage } \\
\text { period }\end{array}$} & \multirow{2}{*}{\multicolumn{2}{|c|}{$\begin{array}{l}\begin{array}{c}\text { Ratio of theophylline } \\
\text { anhydrous form }\end{array} \\
\text { Predicted } \%\end{array}$}} & \multicolumn{4}{|c|}{$\begin{array}{c}\text { Percengate dissolved } \\
\text { at } 45 \mathrm{~min}\end{array}$} \\
\hline & & & \multicolumn{2}{|c|}{ Predicted \% } & \multicolumn{2}{|c|}{ Measured \% } \\
\hline & Mean & S.D. & Mean & S.D. & Mean & S.D. \\
\hline $12 \mathrm{~h}$ & 94.9 & 9.1 & 85.9 & 7.5 & 81.1 & 5.5 \\
\hline $3 d$ & 80.9 & 9.6 & 77.1 & 8.2 & 76.8 & 0.8 \\
\hline $5 \mathrm{~d}$ & 59.0 & 12.9 & 60.5 & 9.8 & 72.7 & 3.7 \\
\hline
\end{tabular}

It was assumed that hydration of theophylline in the tablet initially occurred on the external surface that was in contact with the atmosphere, and the hydrated part gradually progressed to the internal region of the tablet. The external surface of the theophylline tablets stored for $5 \mathrm{~d}$ should be rich in theophylline monohydrated form, whereas the internal region should be rich in anhydrous form. After the beginning of dissolution testing, the theophylline tablets that were stored for $5 \mathrm{~d}$ maintained the tablet shape in the dissolution medium for ca. $2 \mathrm{~min}$. Subsequently, the tablets were swollen and divided into upper and lower parts. After ca. $5 \mathrm{~min}$, the upper part of the tablet broke from the lower part and the internal region was exposed to the dissolution medium. The dissolution rate of theophylline from the internal region should be higher than the external surface. Therefore, the measured dissolution rate was higher than that of the predicted one because of the contribution of rapid theophylline elution from the internal region of the stored theophylline tablet. In theophylline tablets that were stored for $3 \mathrm{~d}$ or less, there should be little to no effect on the difference of the dissolution rate because the tablets did not disintegrate in this manner during the dissolution testings.

\section{Conclusion}

This work showed that terahertz spectroscopy was a nondestructive measuring method to quantitatively analyze the ratio of polymorphs in a drug product. Furthermore, it was also demonstrated that terahertz spectroscopy could be applied to predict the dissolution rate of drug product.

There are many theophylline sustained release tablets. The 
techniques of this work would be applicable to these tablets if the spectrum of theophylline don't overlap with the spectra of the other components and has high specificity. There are also several other tablets in which dissolution rates are affected by the polymorphic change of ingredients. If the spectra of these components have high specificity, these techniques would be applicable to the tablets.

We additively investigated an influence of theophylline particle size on these techniques. Theophylline anhydrous form was used as purchased or passed through a $106 \mu \mathrm{m}$ mesh screen, and two types of theophylline tablets were manufactured with the same methods. The predicted ratios of the theophylline anhydrous form were calculated from the $\mathrm{THz}$ spectra of these theophylline tablets using the multivariate calibration model, and the predicted ratios were slightly different from the theoretical values. Furthermore, the percentages dissolved in $45 \mathrm{~min}$ of these two types of tablets were slightly different from each other. Thus, it would be necessary to use theophylline of a similar particle size for manufacturing the tablets for generation of a calibration model and prediction.

THz spectroscopy has two advantages. First, it is a non-destructive measuring method for drug products and has superior potency to XRPD analysis. Second, THz spectroscopy should be more adequate to analyze polymorphism rather than NIR spectroscopy. Thus, an application of $\mathrm{THz}$ spectroscopy to the pharmaceutical PAT tool is expected.

\section{References}

1) Haleblian J. K., J. Pharm. Sci., 64, 1269-1288 (1975).

2) International Conference on Harmonization, "Specifications: Test Procedures and Acceptance Criteria for New Drug Substances and New
Drug Products: Chemical Substances," Ministry of Health, Labour and Welfare, Tokyo, 1999.

3) Byrn S., Pfeiffer R., Ganey M., Hoiberg C., Poochikian G., Pharmaceut. Res., 12, 945-954 (1995).

4) McMahon L. E., Timmins P., Williams A. C., York P., J. Pharm. Sci., 85, $1064-1069$ (1996).

5) Taylor L. S., Langkilde F. W., J. Pharm. Sci., 89, 1342-1353 (2000).

6) Kogermann K., Aaltonen J., Strachan C. J., Pöllänen K., Veski P., Heinämäki J., Yliruusi J., Rantanen J., J. Pharm. Sci., 96, 1802-1820 (2007).

7) Heinz A., Strachan C. J., Gordon K. C., Rades T., J. Pharm. Pharmacol., 61, 971-988 (2009).

8) Strachan C. J., Taday P. F., Newnham D. A., Gordon K. C., Zeitler J. A., Pepper M., Rades T., J. Pharm. Sci., 94, 837-846 (2005).

9) Taday P. F., Bradley I. V., Arnone D. D., Pepper M., J. Pharm. Sci., 92, $831-838(2003)$.

10) Zeitler J. A., Newnham D. A., Taday P. F., Threlfall T. L., Lancaster R. W., Berg R. W., Strachan C. J., Pepper M., Gordon K. C., Rades T., J. Pharm. Sci., 95, 2486-2498 (2006).

11) Zeitler J. A., Kogermann K., Rantanen J., Rades T., Taday P. F., Pepper M., Aaltonen J., Strachan C. J., Int. J. Pharm., 334, 78-84 (2007).

12) Zeitler J. A., Taday P. F., Pepper M., Rades T., J. Pharm. Sci., 96, 2703-2709 (2007)

13) Suzuki E., Shimomura K., Sekiguchi K., Chem. Pharm. Bull., 37, 493-497 (1989).

14) Donoso M., Ghaly E. S., Pharm. Dev. Technol., 9, 247-263 (2004).

15) Freitas M. P., Sabadin A., Silva L. M., Giannotti F. M., do Couto D. A., Tonhi E., Medeiros R. S., Coco G. L., Russo V. F., Martins J. A., J. Pharm. Biomed. Anal., 39, 17-21 (2005).

16) Otsuka M., Tanabe H., Osaki K., Otsuka K., Ozaki Y., J. Pharm. Sci., 96, 788-801 (2007).

17) Ono M., Tozuka Y., Oguchi T., Yamamoto K., Chem. Pharm. Bull., 49, 1526-1530 (2001)

18) Liu H. B., Chen Y., Zhang X. C., J. Pharm. Sci., 96, 927-934 (2007).

19) International Conference on Harmonization, "Validation of Analytical Procedures: Text and Methodology," Ministry of Health, Labour and Welfare, Tokyo, 1994. 